\title{
Jamming and critical outlet size in the discharge of a two-dimensional silo
}

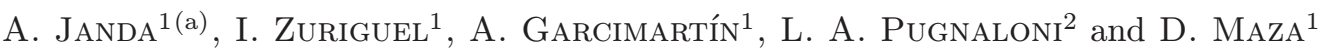 \\ ${ }^{1}$ Departamento de Física y Matemática Aplicada, Universidad de Navarra - 31080 Pamplona, Spain, EU \\ ${ }^{2}$ Instituto de Física de Líquidos y Sistemas Biológicos (CONICET-UNLP-CIC) - Casilla de correo 565, \\ 1900 La Plata, Argentina
}

received 20 June 2008; accepted in final form 13 October 2008

published online 12 November 2008

PACS 47.57.Gc-Granular flow

PACS 45.70.Vn - Granular models of complex systems; traffic flow

\begin{abstract}
We present an experimental study of jamming in the discharge of grains through an opening in a two-dimensional silo. For a wide range of outlet sizes, we obtain the size distribution of avalanche defined as the number of grains that fall between two consecutive jams. From these distributions, we obtain the probability that the silo jams before $N$ particles pass through the orifice. Then a simple model of arch formation is proposed that predicts the shape of the jamming probability function and reveals that it does not exist a critical size of the orifice above which there is not jamming.
\end{abstract}

Copyright (C) EPLA, 2008

Introduction. - Materials in granular form are widely used, having a great importance in the chemical, food, agricultural and pharmaceutical industries [1]. Nevertheless, due to the complex nature of interparticle interactions, the global dynamics of a granular material is not well understood yet [2]. For instance, the flow of grains through an orifice is arrested if the size of the outlet is not large enough. Such a jam entails a complete and permanent halt of the flow. In the outpouring of grains, jamming occurs due to the formation of an arch at the outlet.

Arching is one the most important features of granular materials. Indeed, it has been proposed that arches are responsible for the nonuniform propagation of forces [3] and changes in the packing fraction [4]. An arch is defined as a structure consisting of mutually stabilized particles. If one particle from the arch is removed, the structure collapses under the effect of gravity. In two dimensions, arching has been studied experimentally $[5,6]$, reproduced by numerical simulations [7,8], and analyzed with theories [9]. Let us remark that it could be difficult to establish a straightforward equivalence between the concept of jamming in the discharge of a silo and the notion of jamming as a phase transition introduced by Liu and Nagel [10].

In this work, we have studied the jamming events that appear in the discharge of particles through an opening from a two-dimensional (2D) silo. After a jam, an input

\footnotetext{
(a)E-mail: ajandaga@alumni .unav .es
}

of energy (blowing, shaking or tapping) is necessary to break the blocking arch and restart the flow. Then, the grains fall until a new arch is formed. The size distribution of avalanches (defined as the number of particles fallen between two successive jams) for fixed orifice sizes is obtained. Besides, the relationship between the mean avalanche size and the diameter of the outlet is studied.

Currently, a number of studies propose probabilistic models to explain the avalanche size distribution in silo drainage [11-13]. A common feature of these models is the assumption that each particle passes through the outlet orifice without getting jammed with a probability $p$ that is not influenced by its neighbors. Although the concept is sound, in the sense that the correct shape of the avalanche size distribution is obtained, the value of $p$ is still a fitting parameter. More recently, $p$ was considered in a slightly different process [14] as a function of the packing fraction. In this work, we compare the experimental data obtained for the $2 \mathrm{D}$ case with the predictions of these models and discuss the implications of considering a probability $p$ that depends on the dimensionality of the problem.

This paper is organized as follows. First, we provide a detailed description of the experimental setup. Then, the experimental results of the jamming probability and the mean avalanche size are discussed. Later on, we expound the predictions of a probabilistic model. Finally, some conclusions are drawn about the existence of a critical size of the orifice beyond which jamming has a null probability. 


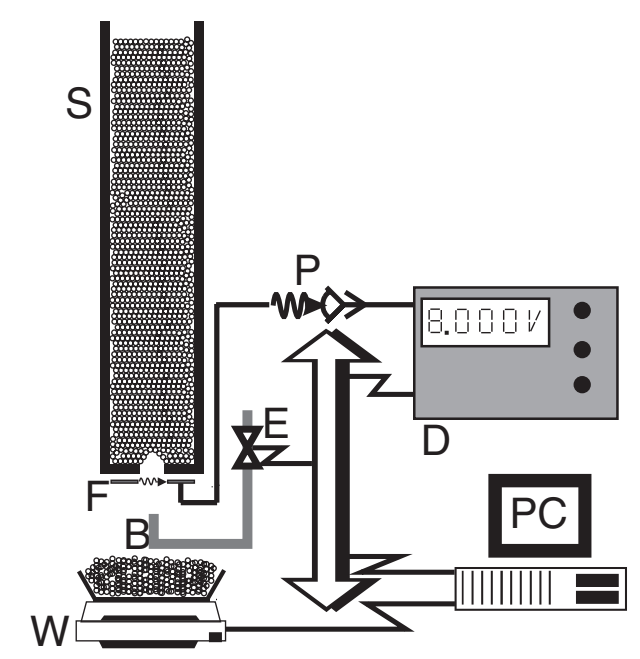

(a)

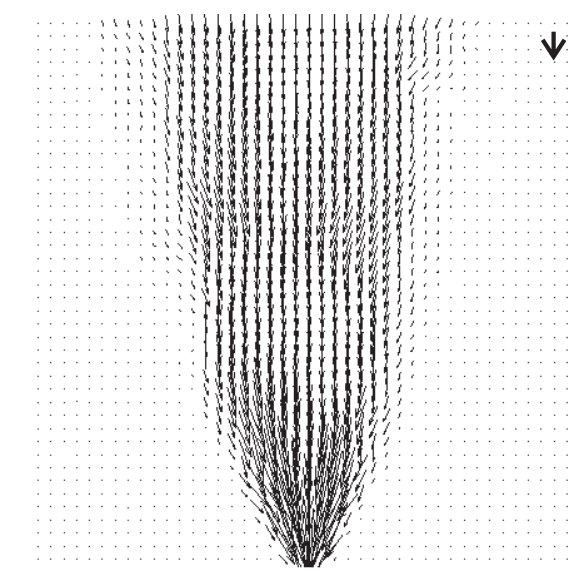

(b)

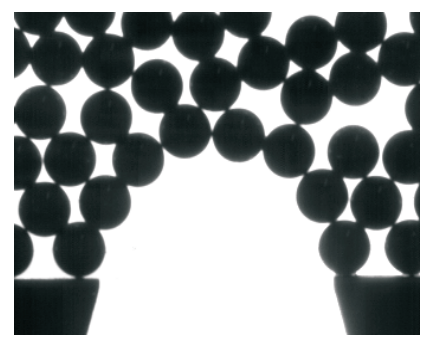

(c)

Fig. 1: (a) Sketch of the experimental setup. S, silo; W, electronic scales; B, blower; E, electrovalve; F, optical fiber; P, photoelectric sensor; D, digitizer; PC, computer. (b) Mean velocity field during the discharge of the $2 \mathrm{D}$ silo in a region near the outlet for $D=5$.0. It has been obtained with a PIV technique. The plot shows the averaging of 50 instantaneous velocity field realizations, which last as a whole $0.1 \mathrm{~s}$. The arrow on the top right corner is a scale which corresponds to $10 \mathrm{~m} / \mathrm{s}$. (c) Photograph of an arch formed above the orifice that has arrested the flow of grains.

Experimental setup. - The experimental setup is a 2D silo consisting of two glass plates between which spherical beads are poured (fig. 1a). Two stainless-steel strips of $1.1 \mathrm{~mm}$ thickness are sandwiched between the glass plates, at the sides, so that they confine the beads in the gap between the plates. The space left between the two strips -i.e. the width of the silo- is about 200 times the diameter of the particles. Since the width is larger than 30 bead diameters it can be stated that the lateral walls do not influence the jamming probability [15]. The granular material used to fill the silo are monodisperse spherical stainless steel beads with mass $4.00 \pm 0.01 \mathrm{mg}$ and diameter $1.00 \pm 0.01 \mathrm{~mm}$. As the separation between the two glass plates is $1.1 \mathrm{~mm}$, the particles can only arrange themselves in a single layer. The flat bottom of the silo is formed by two facing metal pieces, so that their edges fix the outlet slot size $D_{o}$. These two pieces are blade-shaped and widen downwards (see the photograph in fig. 1c). This shape prevents the formation of arches leaning on the walls of the very orifice. The aperture of the slot was measured with a precision that in all the cases was better than $0.06 \mathrm{~mm}$. The control parameter is the dimensionless size of the outlet $D$, defined as the ratio between the width of the opening $D_{o}$ and the diameter of the particles $d$, i.e. $D \equiv D_{o} / d$. When the width of the outlet is large enough, the beads pour freely from the silo due to gravity. However, if the size of the orifice does not exceed a few bead diameters, the flow is arrested due to the formation of an arch (fig. 1c). An optical sensor beneath the orifice detects whether or not particles are flowing.

When an arch is formed at the outlet arresting the flow, we wait for about five seconds to ensure that the arch is stable. Then the flow is resumed breaking the arch by means of a jet of pressurized air from beneath aimed at the orifice. This procedure has been chosen because of its high reproducibility and to preclude changes in the packing fraction of the granular material. In industry, other practices are often put to use, such as vibrating the silo or hitting the walls, but they may change the volume fraction of the granular material through the whole silo [4]. The air gust is controlled by opening an electrovalve, which is driven by a switch that is in turn commanded from a PC. In a previous work [16] it has been shown in a $3 \mathrm{D}$ silo that the duration of the air jet and its pressure do not affect significantly the results. Therefore, as in ref. [16], the pressure of the air is kept at $4.0 \pm 0.5 \mathrm{~atm}$ and it lasts $0.4 \pm 0.1 \mathrm{~s}$. The grains that fall from the silo are collected in a container on top of an electronic scales (model AND GX-400) whose resolution is $1 \mathrm{mg}$. Since the mass of a single bead is $4.00 \pm 0.01 \mathrm{mg}$, this resolution enabled the detection of a single grain. Dividing the collected mass by the weight of one grain, we determine the number of grains fallen between two successive jams. These data are stored in a computer for further analysis. The silo was filled with the help of a hopper placed on top of it so as the grains are poured in a distributed way along the whole width of the silo. Typically, more than $10^{5}$ beads were needed to fill the silo, which is refilled from time to time in order to keep the level of the material above three times the width of the silo. In this way, the pressure at the bottom of the silo is practically unaltered over the course of the experiment so that, for a given orifice diameter, the mean flow rate of grains remains constant [17]. Figure 1b shows the mean velocity field of the particles in a region near 


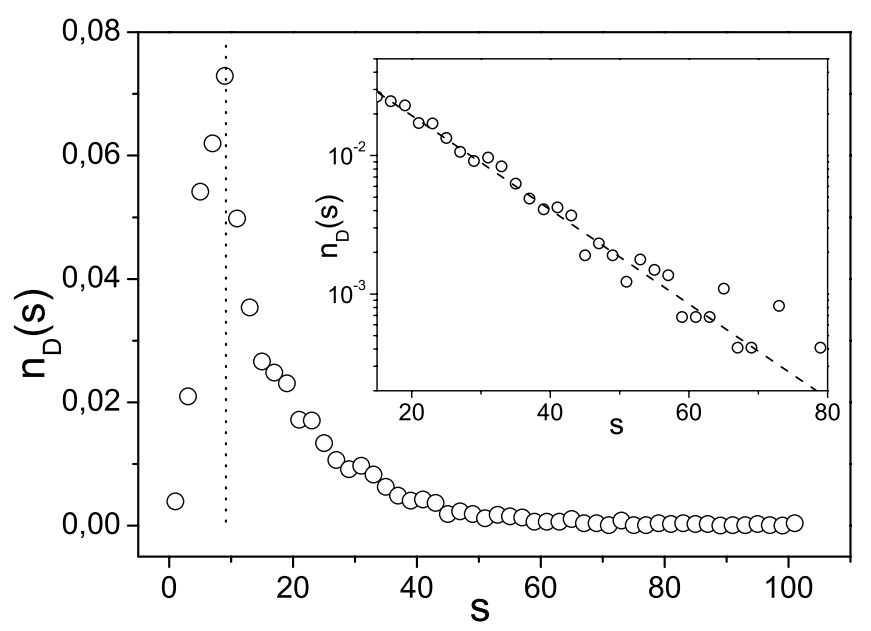

Fig. 2: Histogram of the number of grains $s$ in an avalanche. Data correspond to $D=2.17$. The vertical dashed line indicates the value of the mode. Inset: the same histogram in semilogarithmic scale.

the orifice during an avalanche. Above the orifice particles move with velocities preferentially oriented in the vertical direction, although there are stagnant particles at the bottom corners. The shape of the region where particles are moving is approximately parabolic.

Experimental results. - We have measured the size of the avalanches for $D$ ranging from 1.53 to 5.63. Figure 2 shows the avalanche size distribution $n_{D}(s)$, i.e. the probability of obtaining an avalanche of $s$ grains, for a particular diameter. In order to calculate the histograms, we have measured about 3000 avalanches for each value of $D$. Two different regions can be seen in the histogram. On the left side, for avalanches smaller than the mode, the probability grows with $s$. As explained in ref. [16], this feature is not well understood, and besides it is sensitive to the method used to restart the flow. On the right side of the histogram, the number of avalanches larger than the mode decreases exponentially with $s$ (see the inset in fig. 2). The exponential decay means that the phenomenon is ruled by a characteristic parameter. This allows us the collapse of the histograms into a single plot for all values of $D$ by using the rescaled avalanche size, i.e. $s^{*}=s /\langle s\rangle$, where $\langle s\rangle$ is the mean avalanche size (fig. 3). Interestingly, the same exponential decay of the avalanche size is also found in the discharge of a $3 \mathrm{D}$ silo $[12,16]$.

Let us now define the jamming probability $J$ as the probability that the flow gets arrested before $N$ beads fall. The jamming probability depends on $N$ and on the outlet size $D$. One can write

$$
J(N, D) \equiv 1-\sum_{s=N}^{\infty} n_{D}(s),
$$

which is the probability that the avalanche is smaller than $N$ for a given $D$. If we fix $N$ at some particular value, we can evaluate $J_{N}(D)$ from the data of the avalanche sizes

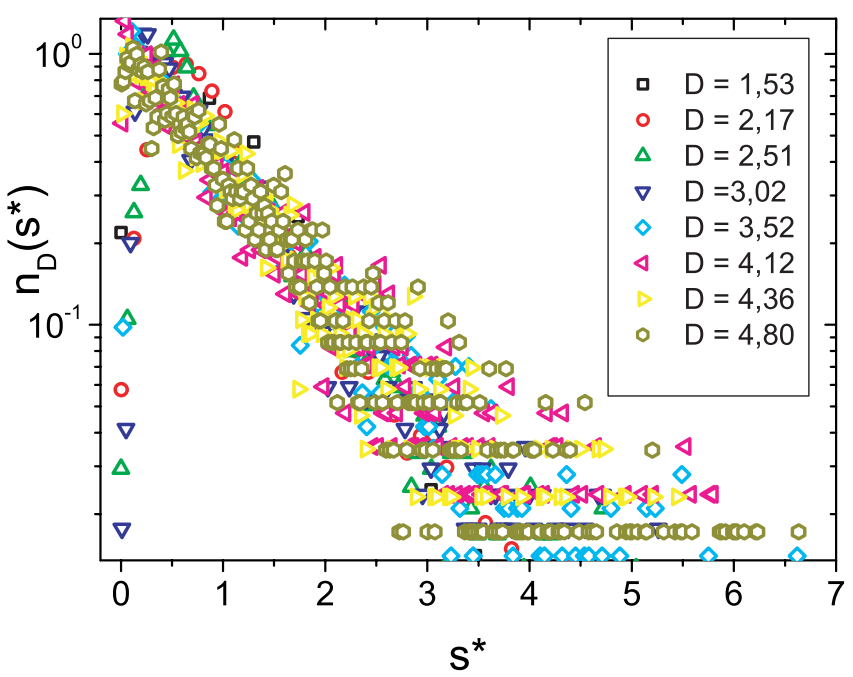

Fig. 3: (Color online) Rescaled histograms for different sizes of the exit orifice $(1.53 \leqslant D \leqslant 4.8)$, as indicated in the legend.

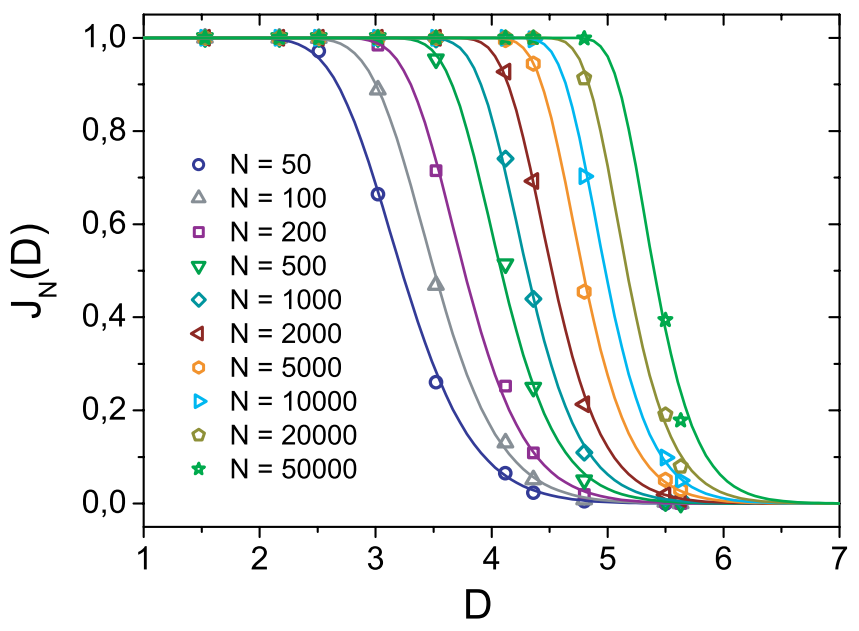

Fig. 4: (Color online) The jamming probability $J_{N}$ as function of $D$. Each set of symbols corresponds to a different value of $N$ ranging from 50 to 50000, as indicated in the legend. Solid lines are plots of the jamming probability given by eq. (7) (see text for details).

corresponding to all the explored values of $D$. In fig. 4 we plot $J_{N}(D)$ for different values of $N$ in a range of outlet sizes spanning from $D=1.53$ to $D=5.63$. (Lines correspond to a model that will be presented in the next section.) For small values of $D$ (such as $D \leqslant 2.5$ ), the value of $J$ is almost one, which means that the probability that the flow gets jammed before $N$ beads have fallen is very high. However, for large values of $D($ e.g., $D \geqslant 6)$, it is very unlikely that the outpouring of grains gets arrested, $J$ being almost zero. Similar results have been found in a two-dimensional hopper filled with disks $[5,11]$.

The shape of fig. 4 suggests that $J_{N}(D)$ could converge to a step function as $N$ increases. If this is so, a critical diameter $D_{c}$ could be introduced above which the probability of jam would be strictly zero. This means that if we calculate the jamming probability for $N \rightarrow \infty$, we would 


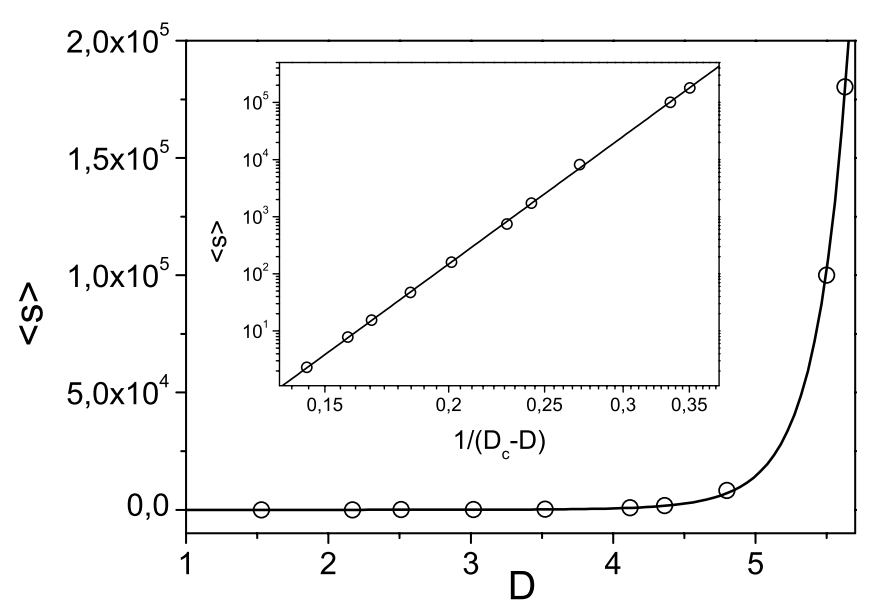

Fig. 5: Experimental results of the mean avalanche size vs. D. The solid line corresponds to the fit with the power law divergence presented in eq. (2). The fitting parameters are: $D_{c}=8.5 \pm 0.1, C=1.1 \times 10^{11} \pm 0.1 \times 10^{11}$ and $\gamma=12.7 \pm 0.1$. In the inset the logarithmic plot of $\langle s\rangle$ vs. $1 /\left(D_{c}-D\right)$ shows the goodness of the fit.

obtain $J_{\infty}=1$ for all $D<D_{c}$, i.e, that the outpouring of beads will be arrested in a finite time for $D<D_{c}$. Yet if $D \geqslant D_{c}$ the orifice would never get blocked, so $J_{\infty}=0$. This would point at the existence of a critical point separating two phases in the discharge process. This idea was supported in ref. [16] by reporting a divergence of the mean avalanche size as $D$ approaches a certain value $D_{c}$ in a $3 \mathrm{D}$ silo. The power law divergence proposed for the $3 \mathrm{D}$ experimental data, namely

$$
\langle s\rangle=\frac{C}{\left(D_{c}-D\right)^{\gamma}}
$$

is found to be also valid for the experimental results in $2 \mathrm{D}$ that are presented in this work (fig. 5). The values of the fitting parameters for $2 \mathrm{D}$ are $D_{c}=8.5 \pm 0.1$, $C=1.1 \times 10^{11} \pm 0.1 \times 10^{11}$ and $\gamma=12.7 \pm 0.1$. It should be noticed that the critical diameter is too far from the values of $D$ where experimental data can be obtained in a reasonable amount of time.

In a recent work, To [11] has shown that for a $2 \mathrm{D}$ hopper the mean avalanche size as a function of the outlet size can be fitted by the aforementioned power law divergency (2), but also by an exponential square $\left(C^{\prime} \exp \left[\gamma^{\prime} D^{2}\right]\right)$ and an exponential reciprocal $\left(C^{\prime \prime} \exp \left[\gamma^{\prime \prime} /\left(D_{c}-D\right)\right]\right)$ with approximately the same accuracy. The fact that the data can be equally well fitted with different expressions, diverging and non-diverging, implies that the existence of a critical diameter is not clear. As in [11], our data can be well fitted by the non-diverging exponential-square function. In fig. 6 , we plot $\ln (\langle s\rangle+1)$ vs. $D^{2}$ and obtain a straight line. The possibility of fitting the mean avalanche size using diverging and non-diverging expressions has also been analyzed numerically [18].

Let us remark, however, that none of these fitting laws are derived from a model of the jamming process. In what

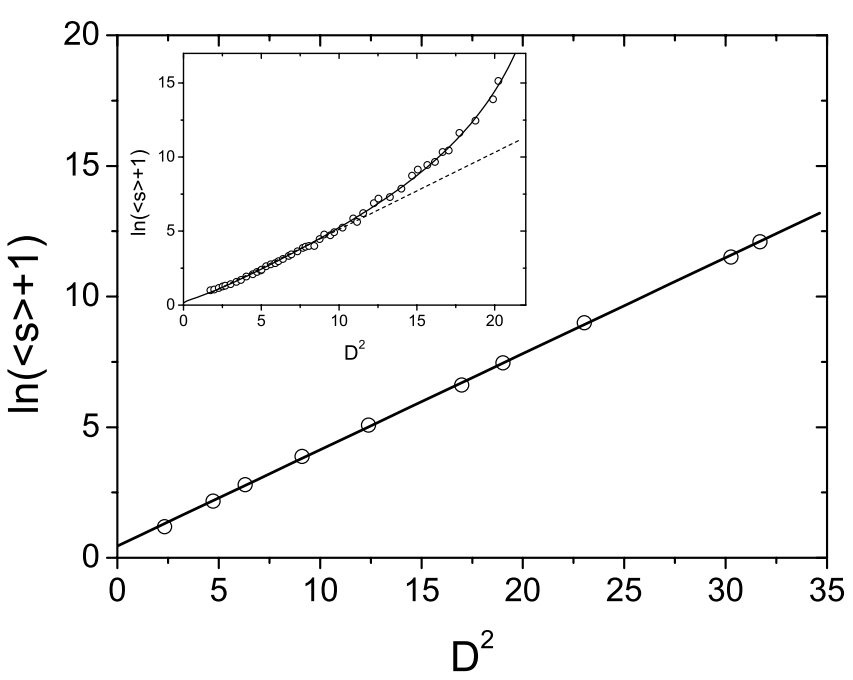

Fig. 6: Plot of $\ln (\langle s\rangle+1)$ as function of $D^{2}$ for the $2 \mathrm{D}$ silo. The solid line is a linear fit. Inset: the same plot as in the main graph with the data from a $3 \mathrm{D}$ silo. The dashed line is a linear fit taking only the data for small $D$. The solid line corresponds to the power law divergency from ref. [16].

follows, we will propose an expression for $\langle s\rangle$ that depends on $D$ and can be obtained from a probabilistic model. This interpretation will be shown to suggest that the correct expression to represent the mean avalanche size for the $2 \mathrm{D}$ case should be the exponential-square function.

Arching and avalanche size distribution. - One of the most puzzling questions about jamming events involves the relationship between the avalanche size distribution and the arching process. A careful inspection of the moving particles near the outlet slot suggests that arch formation is a local process. Without considering the details of the process by which an arch is generated, the mean avalanche is undoubtedly related to the length of the slot through the width that an arch can span just above it (see, for instance, fig. 1c). In the preceding section we showed that the mean avalanche size $\langle s\rangle$ rescales all the histograms in a single one. Hence, a probabilistic model linking the jamming probability with the size of the orifice could be obtained if $\langle s\rangle$ can be related to the probability $\tilde{p}$ that an arch stops the flow.

In a previous work [16], a simple model was proposed to describe the exponential decay of $n_{D}(s)$ for large $s$ introducing the probability $p$ for a particle to pass through the orifice without forming a blocking arch with its neighbors:

$$
n_{D}(s)=p^{s}(1-p) .
$$

Assuming that $p$ remains constant for all the beads during the avalanche it is possible to relate $p$ and the mean avalanche size $\langle s\rangle$. Hence, the first moment of the distribution described by eq. (3) can be written as

$$
\langle s\rangle=\frac{p}{1-p} .
$$


In the study of the clogging of a sieve during filtration of particles suspended in a gel, Roussel et al. [14] estimated the probability $\tilde{p}$ that a number $\eta^{\prime}$ of spheres meet at the time of passing through an orifice, which is a necessary condition for the formation of an arch. Then, provided that $\tilde{p}$ is not only proportional to the probability that a particle meets $\eta^{\prime}-1$ others at the time of passing through the orifice, but also proportional to the probability of forming an arch, we can write $p=1-\tilde{p}$.

Extending this idea to the $2 \mathrm{D}$ silo configuration, we can estimate $p$ from the probability $\tilde{p}$ of finding an arch of $\eta$ grains obtained in computer simulations (note the change of variable from $\eta^{\prime}$ in $3 \mathrm{D}$ to $\eta$ in $2 \mathrm{D}$ : in general, the variable describing the number of beads constituting an arch will have different features depending on the dimensions). Results from two different simulation techniques $[7,8]$ used to model disks in a $2 \mathrm{D}$ container show that $\tilde{p}=A \exp \left[-B \eta^{2}\right]$. The average number of grains that typically block the orifice should be a smooth function of the width of the slot. If one assumes the simplest hypothesis, namely, that $\eta$ grows linearly with $D$, i.e. $\eta=\eta_{0} D$, then eq. (4) can be written as

$$
\langle s\rangle=A^{-1} \exp \left[B\left(\eta_{0} D\right)^{2}\right]-1 .
$$

Equation (5) reveals that the arch size distribution probability is coherent with an exponential-square dependence of the mean avalanche size on the outlet diameter. Interestingly, this function is one of the several expressions that To [11] showed to fit his data for a 2D hopper. From fig. 6 we observe that the plot of $\ln (\langle s\rangle+1)$ vs. $D^{2}$ does yield a straight line, in agreement with eq. (5). Thus the probabilistic explanation of the arching process is able to describe the exponential-square growth of $\langle s\rangle$ with $D$. This interpretation also predicts that no critical diameter exists, since the mean avalanche size remains finite for any finite value of $D$.

From eqs. (1), (3) and (4), we can write for the jamming probability

$$
\begin{aligned}
J_{N}(D)= & 1-p^{N}=1-\left(\frac{\langle s\rangle}{1+\langle s\rangle}\right)^{N}= \\
& 1-\exp \left[N \ln \left(1+\langle s\rangle^{-1}\right)\right]_{s \gg 1} 1-\exp [-N /\langle s\rangle] .
\end{aligned}
$$

Inserting (5) into (6) we obtain for $s \gg 1$ :

$$
J_{N}(D)=1-\exp \left[-N A e^{-B\left(\eta_{0} D\right)^{2}}\right]
$$

The solid lines in fig. 4 correspond to the prediction of eq. (7) with the parameters $A$ and $B \eta_{0}^{2}$ obtained from the fit of (5) to the data shown in fig. 6. Equation (7) indicates that the plot of $J_{N}(D)$ as a function of the rescaled variable $x=N A e^{-B\left(\eta_{0} D\right)^{2}}$ for different values of $N$ must result in a single curve. In fig. 7, where $J(x)$ is plotted for several values of $N$, it can be seen that this is indeed the case. Let us stress that the collapsed data do not lead to a step function, in agreement with the fact that eqs. (5) and (7) do not contain a critical size of the orifice above which there is not jamming.

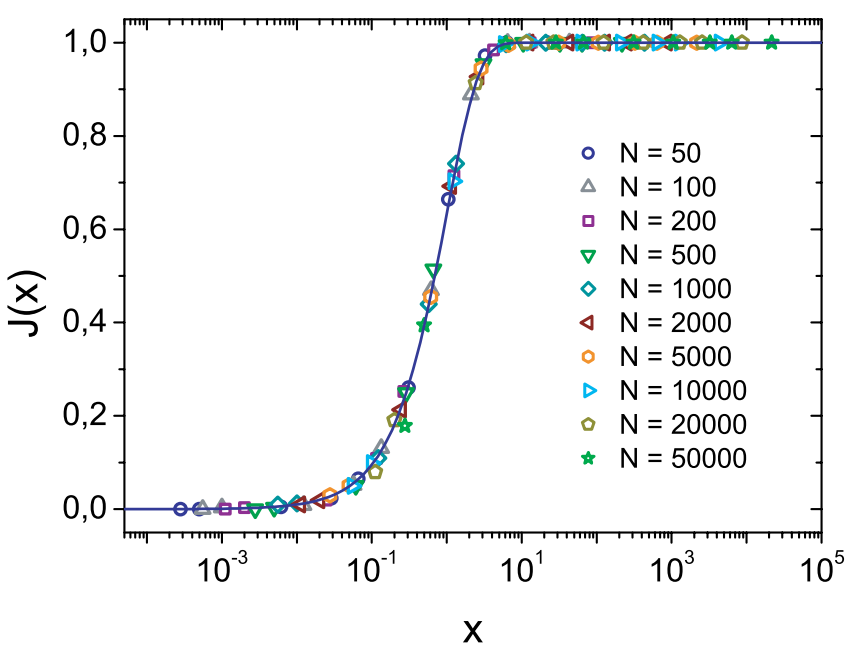

Fig. 7: (Color online) The jamming probability $J$ as a function of the rescaled variable $x=N A e^{-B\left(\eta_{0} D\right)^{2}}$. The different symbols correspond to the different values of $N$ displayed in the legend. The solid line is the function $1-\exp (-x)$.

In principle it seems reasonable that the analysis carried out for the $2 \mathrm{D}$ silo may be extended to a $3 \mathrm{D}$ case. Roussel et al. estimate that the probability $\tilde{p}$ that a number $\eta^{\prime}$ of spheres meet at the time of passing through an orifice in $3 \mathrm{D}$ can be written as $\tilde{p}=A^{\prime} \exp \left[-B^{\prime} \eta^{\prime}\right]$. This is in good agreement with other results of arch formation in 3D simulations [19] and 3D deposits of colloidal particles scanned with confocal microscopy [6]. On the contrary it is remarkable that the expression for $\tilde{p}$ in $3 \mathrm{D}$ is different from the one obtained in 2D numerical simulations. Assuming that the number of grains needed to block an orifice in a $3 \mathrm{D}$ container is proportional to the opening area, i.e. $\eta^{\prime}=$ $\eta_{0}^{\prime} D^{2}$, and recalling that $p=1-\tilde{p}$, the mean avalanche size can be written as

$$
\langle s\rangle_{3 D}=A^{\prime-1} \exp \left[B^{\prime} \eta_{0}^{\prime} D^{2}\right]-1 .
$$

According to this notion, the plot of $\ln \left(\langle s\rangle_{3 D}+1\right) v s$. $D^{2}$ should yield a straight line. As can be seen in the inset of fig. 6 for the 3D silo, this is not the case. The experimental data displays a growth considerably faster than the exponential square predicted by eq. (8). Incidentally, it seems rather counterintuitive that the prediction for the dependence of $\langle s\rangle_{3 D}$ on $D$ is the same as for two dimensions (compare eqs. (8) and (5)). The origin of this discrepancy between the model and the experimental results in the $3 \mathrm{D}$ case may be either in the assumption that in three dimensions $\tilde{p}=A^{\prime} \exp \left[-B^{\prime} \eta^{\prime}\right]$ or in the assumption that $\eta^{\prime}=\eta_{0}^{\prime} D^{2}$. Interestingly, it should be mentioned that for the $3 \mathrm{D}$ case a fit of the number of grains with an exponential of $D^{3}$ is not satisfactory either. A more detailed analysis must be carried out to clarify the role of the dimensionality and the existence or not of a critical diameter.

Conclusions. - In this paper, we have presented an experimental study of the jamming during the discharge 
of spherical particles from a two-dimensional silo. The avalanche size distribution $n_{D}(s)$ shows an exponential tail for avalanches larger than the mode, in coincidence with results from 3D silos. We calculate the jamming probability $J_{N}(D)$ for different $N$ as a function of the orifice diameter. In order to relate this probability to the mean avalanche size we extended a previous model [12] taking into account the typical size of the arch that blocks the outlet orifice. Under this approximation the predictions of the model fit the whole range of diameters studied. An important consequence of this estimation is that no critical opening size exists beyond which there is not jamming. This model predicts the same functional form for the $3 \mathrm{D}$ case. However, the experimental data for this situation display a much faster growth of the mean avalanche size, and it has been shown that this growth can be fitted considering the existence of a critical radius [16]. A detailed study of the structure of the blocking arches in $2 \mathrm{D}$ and $3 \mathrm{D}$ silos may shed light on this issue.

$$
* * *
$$

We thank A. SAntos (Universidad de Extremadura, Spain) for discussions and suggestions, and A. DE LA Torre, M. PAstor and C. Mankoc for their help. This work has been financially supported by Projects FIS 200503881 (Spanish Government), A/9903/07 (AECI) and PIUNA (Universidad de Navarra). AJ thanks Fundación Ramón Areces for a scholarship. LAP acknowledges financial support from CONICET (Argentina).

\section{REFERENCES}

[1] Nedderman R. M., Statics and Kinematics of Granular Materials (Cambridge University Press, London) 1992.
[2] Jaeger H. M., Nagel S. R. and Behringer R. P., Rev. Mod. Phys., 68 (1996) 1259.

[3] O'Hern C. S., Langer S. A., Liu A. J. and Nagel S. R., Phys. Rev. Lett., 86 (2001) 111.

[4] Nowak E. R., Knight J. B., Ben-Naim E., Jaeger H. M. and NAGEL S. R., Phys. Rev. E, 57 (1998) 1971.

[5] To K., Lai P. Y. and PaK H. K., Phys. Rev. Lett., 86 (2001) 71.

[6] Jekins M., PhD Thesis (The University of Edinburgh School of Physics, Edinburgh) 2006.

[7] Pugnaloni L. A. and Baker G. C., Physica A, 337 (2004) 428.

[8] Arevalo R., Maza D. and Pugnaloni L. A., Phys. Rev. E, 74 (2006) 021303.

[9] Drescher A., Waters A. J. and Rhoades C. A., Powder Technol., 84 (1995) 177.

[10] LiU A. J. and NAgel S. R., Nature, 21 (1998) 396.

[11] To K., Phys. Rev. E, 71 (2005) 060301(R).

[12] Zuriguel I., Pugnaloni L. A., Garcimartín A. and Maza D., Phys. Rev. E, 68 (2003) 30301(R).

[13] Santos A., private communication.

[14] Roussel N., Nguyen T. L. H. and Coussot P., Phys. Rev. Lett., 98 (2007) 114502.

[15] Hirshfeld D. and Rapaport D. C., Eur. Phys. J. E, 4 (2001) 193.

[16] Zuriguel I., Garcimartín A., Maza D., Pugnaloni L. A. and Pastor J. M., Phys. Rev. E, 71 (2005) 051303.

[17] Mankoc C., Janda A., ArÉvalo R., Pastor J. M., Zuriguel I., Garcimartín A. and Maza D., Granular Matter, 9 (2007) 407.

[18] PÉrez G., Pramana-J. Phys., 70 (2008) 989.

[19] Although ref. [7] shows that the probability of finding a 3D arch of $\eta^{\prime}$ spheres decays as a power law in hybrid Monte Carlo followed by sequential deposition simulations, recent revisions of the arch detection algorithms yield an exponential decay (BARKER G. C., private communication). 